\title{
Mitochondria and diabetes
}

\author{
Gerasimos Siasos $^{1}$, Stavroula A. Paschou ${ }^{1,2}$, Dimitrios Tousoulis ${ }^{1}$ \\ ${ }^{1}$ Department of Cardiology, Hippokration Hospital, ${ }^{2}$ Division of Endocrinology and Diabetes, "Aghia Sophia" Hospital, Medical School, National \\ and Kapodistrian University of Athens, Athens, Greece \\ Correspondence to: Gerasimos Siasos. Professor, Department of Cardiology, Hippokration Hospital, Medical School, National and Kapodistrian \\ University of Athens, 11527 Athens, Greece. Email: gsiasos@med.uoa.gr. \\ Provenance and Peer Review: This article was commissioned by the Editorial Office, Annals of Translational Medicine. The article did not undergo \\ external peer review.
}

Submitted Feb 12, 2020. Accepted for publication Feb 29, 2020.

doi: $10.21037 / \mathrm{atm} .2020 .03 .15$

View this article at: http://dx.doi.org/10.21037/atm.2020.03.15

It is estimated that about 1 of 11 persons in the Western world are currently suffering from type 2 diabetes mellitus (T2DM). Moreover, a large percentage of individuals having the disease remain undiagnosed (1). The main cause of this phenomenon is the increase in the percentage of central obesity, due to global aging of the population, excessive energy intake, decreased energy expenditure and chronic stress. Central obesity leads to the secretion of certain cytokines and adipokines from the excess adipose tissue that promote systemic low-grade inflammation and insulin resistance (2). The initial response of pancreatic beta cells is the secretion of excessive amounts of insulin, as they have to compensate insulin resistance and to maintain glucose homeostasis $(3,4)$. Only a part of individuals with central obesity will ultimately develop the disease, as T2DM presentation requires a certain genetic predisposition of dysfunction of the pancreatic beta cells (2).

T2DM represents an important risk factor for the presentation of cardiovascular disease (CVD) and death from CVD. In fact, about $68 \%$ of people over 65 years of age with T2DM die because of some type of CVD, while $16 \%$ die because of stroke $(1,5)$. In other words, adults with T2DM are 2 to 4 times more likely to die from CVD than adults without T2DM. Large epidemiological studies suggest that people with T2DM present the same risk for CVD as non-diabetic's individuals who are older by 15 years $(1,5,6)$. Costs for healthcare are very high for these patients as well (7). All these data highlight the importance of further unravelling the pathophysiological pathways of T2DM, in order to reduce the global burden of the disease. In this context, mitochondrial function has gained great interest recently in terms of insulin resistance and beta cells function.

Reactive oxygen species (ROS) are mainly produced within mitochondria, specifically emerged from the mitochondrial electron transport chain (ETC) $(8,9)$. In the case of overnutrition, such as in obesity, a large amount of electrons are supplied into the respiratory chain. Moreover, when physical activity is decreased, the demand for energy and ATP is low. This leads to superoxide formation and increased oxidative stress, as a high proton motive force with a low respiration rate is created. Prolonged oxidative stress directly impacts metabolism and increases insulin resistance in peripheral tissues. Endogenous defects in mitochondrial function can further affect the above and increase oxidative stress and subsequent insulin resistance (9). The excessively oxidative environment increases the number of hypertrophic adipocytes. Moreover, such an environment alters the activity state of certain enzymes, leading for example to lipid peroxidation or protein carbonylation. This happens either directly or indirectly by post-translational modifications. Cumulatively, the increased oxidative stress alters adipogenesis, the number and amount of adipocytes, as well as their adipokines secretion profile $(4,9)$.

Regarding beta pancreatic cells, these are physiologically able to keep a stable amount of functional mitochondria by complex preventive mechanisms of their fusion with the depolarized ones. The latter are recognized by autophagosomes and fuse with lysosome in order to degrade their content. Moreover, the used mitochondria can still generate ATP in response to glucose for some time, therefore they can stimulate insulin secretion (10). 
In the case of obesity or increased insulin resistance, beta cells are exposed to acute high concentrations of free fatty acids (FFAs). In that case, ROS production is increased and mitochondria depolarize and fragment. Various metabolites are then produced, such as malate and succinate, that stimulate exocytosis and increased basal insulin secretion, resulting in hyperinsulinemia $(9,10)$. Under chronic high increased concentrations of FFAs (lipotoxicity) or/and glucose concentrations (glucotoxicity), ROS production is further increased. This way the number of depolarized mitochondria is increased, while the overall ATP/ADP ratio is decreased, therefore the glucose stimulated insulin secretion is suppressed. Additionally, lysosomes become acidic and the proper clearance of used mitochondria is prevented. These mitochondria accumulate more and more, leading ultimately into apoptosis of the pancreatic beta cells (8-10).

In conclusion, mitochondrial dysfunction is associated with both insulin resistance and beta cells defects that both predispose to the development of T2DM. As T2DM is a real epidemic in the Western World, therapeutic interventions against mitochondrial dysfunction represent an optimistic therapeutic target for experimental and human studies with the hope of treatment or prevention of this common and important disease.

\section{Acknowledgments}

Funding: None.

\section{Footnote}

Conflicts of Interest: GS serves as an unpaid editorial board member of Annals of Translational Medicine from Sep 2018 to Aug 2020. The other authors have no conflicts of interest to declare.

Ethical Statement: The authors are accountable for all aspects of the work in ensuring that questions related to the accuracy or integrity of any part of the work are appropriately investigated and resolved.

Open Access Statement: This is an Open Access article distributed in accordance with the Creative Commons Attribution-NonCommercial-NoDerivs 4.0 International License (CC BY-NC-ND 4.0), which permits the noncommercial replication and distribution of the article with the strict proviso that no changes or edits are made and the original work is properly cited (including links to both the formal publication through the relevant DOI and the license). See: https://creativecommons.org/licenses/by-nc-nd/4.0/.

\section{References}

1. World Health Organization_Diabetes. Available online: https://www.who.int/news-room/fact-sheets/detail/ diabetes

2. Paschou SA, Anagnostis P, Goulis DG. Weight loss for the prevention and treatment of type 2 diabetes. Maturitas 2018;108:A1-2.

3. Rabe K, Lehrke M, Parhofer KG, et al. Adipokines and insulin resistance. Mol Med 2008;14:741-51.

4. Taylor R. Insulin resistance and type 2 diabetes. Diabetes 2012;61:778-9.

5. World Health Organization_Cardiovascular Disease. Available online: https://www.who.int/health-topics/ cardiovascular-diseases

6. Introduction: Standards of Medical Care in Diabetes-2019. Diabetes Care 2019;42:S1-2.

7. McCombie L, Leslie W, Taylor R, et al. Beating type 2 diabetes into remission. BMJ 2017;358:j4030.

8. Siasos G, Tsigkou V, Kosmopoulos M, et al. Mitochondria and cardiovascular diseases-from pathophysiology to treatment. Ann Transl Med 2018;6:256.

9. Masschelin PM, Cox AR, Chernis N, Hartig SM. The Impact of Oxidative Stress on Adipose Tissue Energy Balance. Front Physiol 2020;10:1638.

10. Las G, Oliveira MF, Shirihai OS. Emerging roles of $\beta$-cell mitochondria in type-2-diabetes. Mol Aspects Med 2020;71:100843.
Cite this article as: Siasos G, Paschou SA, Tousoulis D. Mitochondria and diabetes. Ann Transl Med 2020;8(6):262. doi: 10.21037/atm.2020.03.15 